\title{
Author Correction: Casimir spring and dilution in macroscopic cavity optomechanics
}

\author{
J. Mummery, J. M. Pate $\mathbb{D}$, M. Goryachev, R. Y. Chiao, J. E. Sharping and M. E. Tobar (D)
}

Correction to: Nature Physics https://doi.org/10.1038/s41567-020-0975-9, published online 3 August 2020.

In the version of the Article originally published, the gap sizes are calculated from the fundamental microwave resonant frequency using formulas presented in [1]. However, the dimensions of the cavity are outside the region of validity of the lumped circuit model, particularly for gap sizes greater than $0.7 \mu \mathrm{m}$. This issue can be avoided by interpolating between eigenfrequency solutions of finite element electromagnetic simulations with varying geometries, which is known to produce experimentally verifiable results outside the region of validity of analytical models of re-entrant cavities[2,3]. This recalibration was performed using data simulated with COMSOL Multiphysics, and the original and corrected versions of Figs. 2 and 4 are shown below. Additionally, the upper axis of Fig. 3 has been rescaled, as shown below. Further information is available in the Supplementary Information accompanying this amendment. These changes have no effect on the conclusions drawn in the article.

[1] Fujisawa, K. IRE Trans. Microw. Theory Tech. 6, 344 (1958).

[2] McAllister, B. T. \& Tobar, M. E. J. Appl. Phys. 123, 226102 (2018).

[3] Le Floch, J.-M. et al. Rev. Sci. Instrum. 84, 125114 (2013).

Supplementary information is available in the online version of this Amendment.

The changes have been made to the online version of the article.

\section{Additional information}

Supplementary information The online version contains supplementary material available at https://doi.org/10.1038/s41567-021-01454-2.
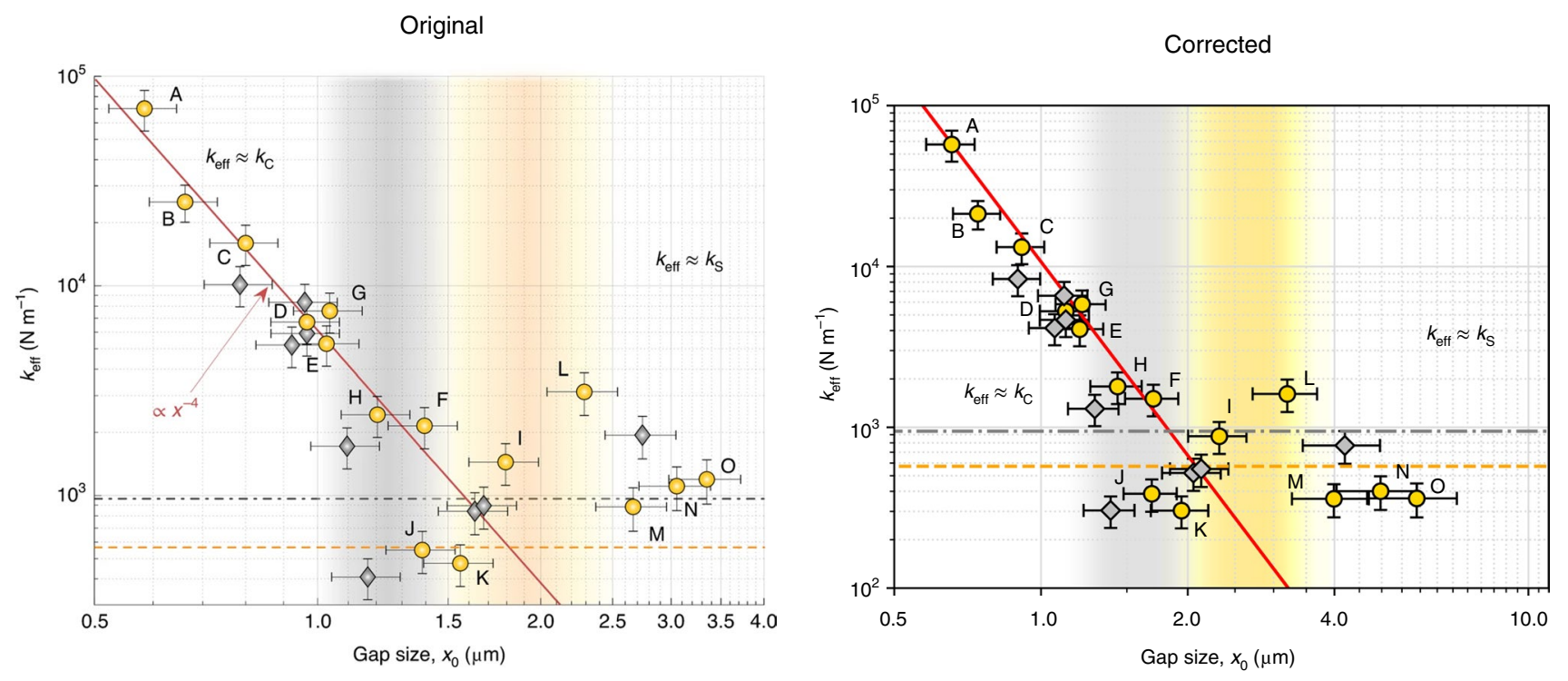

Fig. 2 | Original and Corrected. 

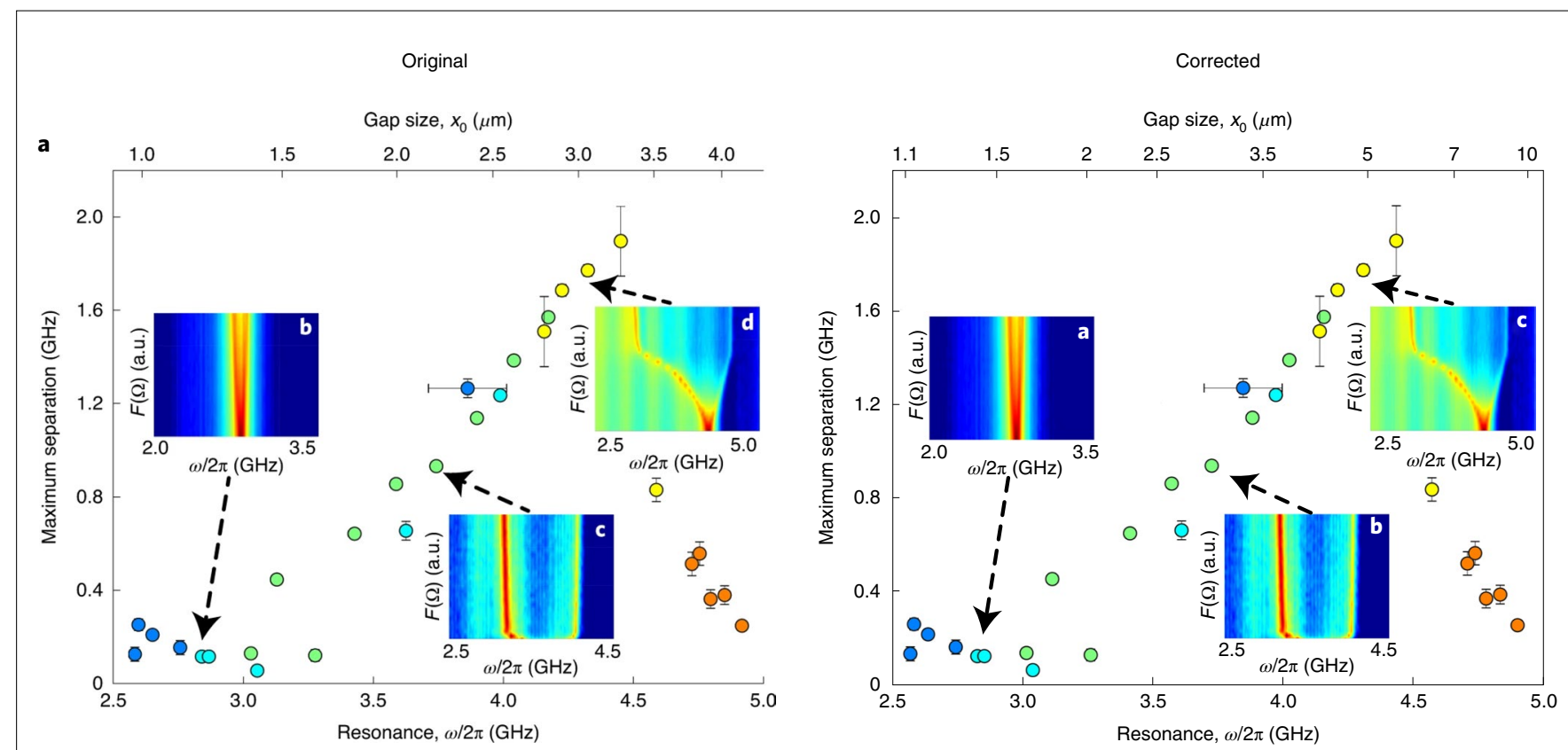

Fig. 3 | Original and Corrected.

Original

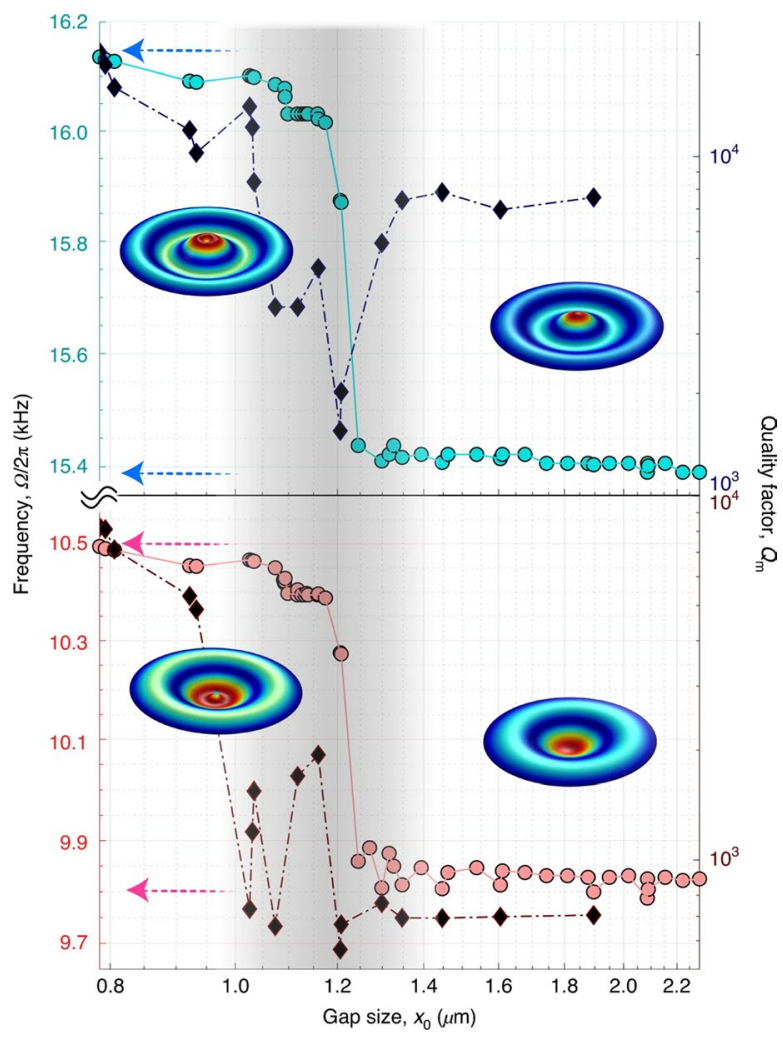

\section{Corrected}

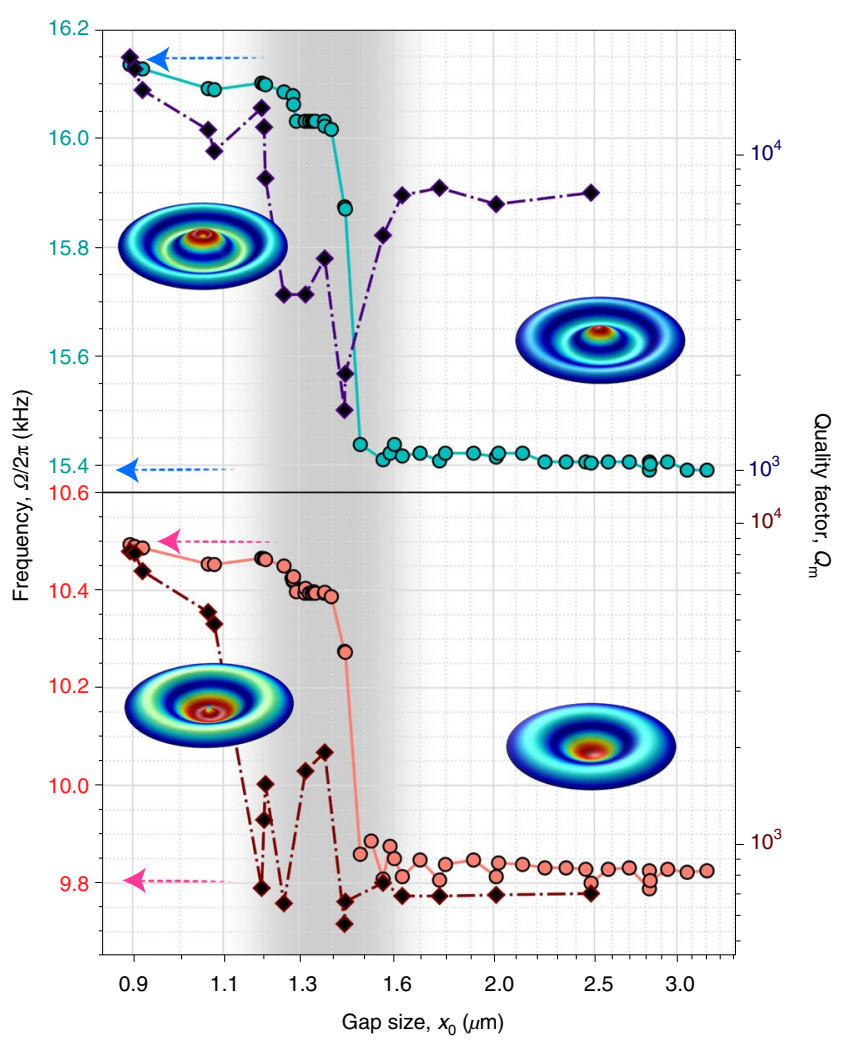

Fig. 4 | Original and Corrected.

Published online: 1 December 2021

https://doi.org/10.1038/s41567-021-01454-2

๑ $\odot$ The Author(s), under exclusive licence to Springer Nature Limited 2021 